\title{
LONGITUDE SURGERY ON GENUS 1 KNOTS
}

\author{
HOWARD LAMBERT ${ }^{1}$
}

\begin{abstract}
Let $l(K)$ be the closed 3-manifold obtained by longitude surgery on the knot manifold $K$. Let $C$ be the cube with holes obtained by removing an open regular neighborhood of a minimal spanning surface in $K$. The main result of this paper is that if $K$ is of genus 1 and the longitude of $K$ is in each term of the lower central series for $\Pi_{1}(C)$, then $l(K)$ is not homeomorphic to the connected sum of $S^{1} \times S^{2}$ and a homotopy 3-sphere. In particular, this implies we cannot obtain the connected sum of $S^{1} \times S^{2}$ and a homotopy 3-sphere by longitude surgery on any pretzel knot of genus 1.
\end{abstract}

Let $K$ be a knot manifold, i.e., $K$ is the complement of an open regular neighborhood of a piecewise linear simple closed curve in $S^{3}$. We assume that $\Pi_{1}(K) \neq Z$ (the knot is really knotted). The longitude $l$ of $K$ is the unique (up to isotopy in $\mathrm{Bd} K$ ) simple closed curve in $\mathrm{Bd} K$ which is homologous to zero in $K$ but bounds no disk in $\mathrm{Bd} K$. It has been conjectured that if we glue a solid torus $T$ to $K$ along the boundary of each in such a way that the meridian of $T$ (a simple closed curve bounding a disk in $T$ but none in $\mathrm{Bd} T$ ) is identified with $l$, we do not obtain $S^{1} \times S^{2}$, or, more generally, we do not obtain a closed 3-manifold which is the connected sum of $S^{1} \times S^{2}$ and a homotopy 3-sphere (denote such a manifold by $\left.\left(S^{1} \times S^{2}\right)^{\prime}\right)$. We refer to the above operation as longitude surgery on $K$ and denote the resulting closed orientable manifold by $l(K)$ (note that $H_{2}(l(K))=Z$ ). In this note we give an algebraic condition which implies that for genus 1 knots,

$$
l(K) \neq\left(S^{1} \times S^{2}\right)^{\prime} .
$$

Louise Moser [6] has shown that $l(K) \neq\left(S^{1} \times S^{2}\right)^{\prime}$ for $K$ a doubled knot, a composite knot or a knot with nontrivial Alexander polynomial.

Let $S$ be an orientable, incompressible [3] surface in $K$ such that $\mathrm{Bd} S$ is $K$ 's longitude. Since such an $S$ always exists for any knot $K$ (see [2]), we assume that the genus of $S$ is smallest possible. Such an $S$ will be referred to

Received by the editors May 20, 1975 and, in revised form,July 12, 1976.

AMS (MOS) subject classifications (1970). Primary 55A25, 57A10; Secondary 55A05, 57A65.

Key words and phrases. Longitude surgery, genus 1 knots, pretzel knots, connected sum of $S^{1} \times S^{2}$ and a homotopy 3 -sphere.

'The author would like to thank Francisco Gonzales-Acuña for several valuable conversations. 
as a minimal spanning surface for $K$ and the genus of $K$ is the genus of $S$. Let $C$ be the cube with holes obtained by removing from $K$ an open regular neighborhood of $S$ in $K$. There is a natural decomposition of $\mathrm{Bd} C$ into three submanifolds $S_{1}, S_{2}$, and $A_{0}$, where $S_{1}, S_{2}$ are copies of $S$, one on either side of $S$, and $A_{0}$ is the annulus which connects $S_{1}$ to $S_{2}$ and its center-line is a longitude of $K$. Let $G$ be a group, $G_{i}$ the $i$ th term in the lower central series and $G_{\omega}=\cap_{i=1}^{\infty} G_{i}$ (see [4] or [5]).

We need two lemmas before proving Theorem 1 .

LEMMA 1. If $l(K)=\left(S^{1} \times S^{2}\right)^{\prime}$, then $K$ contains a properly embedded connected planar surface $X$ such that the number of components of $\operatorname{Bd} X$ is odd, each component of $\mathrm{Bd} X$ is parallel to $K$ 's longitude l, and $X$ is incompressible in $K$.

Proof. Suppose $l(K)=\left(S^{1} \times S^{2}\right)^{\prime}$, that is, longitude surgery on $K$ produces a closed orientable 3-manifold which factors into $S^{1} \times S^{2}$ and a homotopy 3-sphere. Regard the knot manifold $K$ and the attached solid torus $T$ as submanifolds of the closed, orientable manifold $l(K)$. Put $p \times S^{2}$ $\left(\subset S^{1} \times S^{2}, p \in S^{1}\right.$ ) in general position relative to $K$. It follows that we may isotope $p \times S^{2}$ until $T \cap\left(p \times S^{2}\right)$ consists only of meridianal disks of $T$ and, hence, $\left(p \times S^{2}\right) \cap \operatorname{Bd} K$ consists of longitudes of $K$. Since $p \times S^{2}$ does not separate in $l(K)$ and $\left(p \times S^{2}\right)-T$ is connected, the number of components of $\left(p \times S^{2}\right) \cap \mathrm{Bd} K$ is odd [1]. If $X^{\prime}=\left(p \times S^{2}\right) \cap K$ is compressible in $K$, then there would exist a disk $\Delta$ in $K$ such that $\Delta \cap X^{\prime}=\operatorname{Bd} \Delta$ and $\operatorname{Bd} \Delta$ separates some component of $\mathrm{Bd} X^{\prime}$ from another in $X^{\prime}$. We may then remove a small open regular neighborhood of $\mathrm{Bd} \Delta$ from $X^{\prime}$ and fill in the two resulting holes by disjoint parallel copies of $\Delta$. We repeat this process a finite number of times, choosing at each stage the planar surface with an odd number of boundary components. When we cannot go any further, we have the desired planar surface $X$ of the hypothesis.

LEMMA 2. Suppose $M$ is any 3-manifold contained in $S^{3}$ and $X$ is a connected planar surface properly embedded in $M$ such that the number of components of $\mathrm{Bd} X$ is odd and each component of $\mathrm{Bd} X$ lies in an annulus $Y, Y \subset \mathrm{Bd} M$, and is parallel to $Y$ 's center-line. Then the center-line of $Y$ is in $\left(\Pi_{1}(M)\right)_{\omega}$.

Proof. Because the number of components of $\mathrm{Bd} X$ is odd and because each of them is parallel to $Y$ 's center-line $y$, it follows that we can add subannuli $Y_{1}^{\prime}, \ldots, Y_{m}^{\prime}$ of $Y$ to $X$ and adjust the result slightly to form an orientable surface $X^{\prime}$ in $M$ with one boundary component $y$. Let $x_{0}$ be a point in $y$. Then, in $X^{\prime}$, there is a collection of simple closed curves $\alpha_{i}, \beta_{i}$, $i=1, \ldots, m$, having only the one point $x_{0}$ in common and such that each $\alpha_{i}$ is contained in $X$ and separates one boundary component of $Y_{i}^{\prime}$ from the other in $X$ but fails to separate the boundary components of each $Y_{j}^{\prime}, j \neq i$, in $X$, and each $\beta_{i}$ crosses the handle in $X^{\prime}$ formed by $Y_{i}^{\prime}$ once and otherwise $\beta_{i} \cap Y_{j}^{\prime}=\varnothing, \quad i \neq j$. Since $X$ is planar, it follows that $[y]=$ 
$\prod_{i=1}^{m}\left[\alpha_{i}\right]\left[\beta_{i}\right]\left[\alpha_{i}\right]^{-1}\left[\beta_{i}\right]^{-1}$ in $\Pi_{1}\left(X^{\prime}, x_{0}\right)$. Now, in $\left(X^{\prime}, x_{0}\right)$, each loop $\alpha_{i}$ is homotopic to a loop $c_{i}^{\prime} y^{\prime}\left(c_{i}^{\prime}\right)^{-1}$ where $c_{i}^{\prime}$ is a path from $x_{0}$ to one boundary component of $Y_{i}^{\prime}$ and $y^{\prime}$ is a loop which goes once around this boundary component of $Y_{i}^{\prime}$. In $\left(M, x_{0}\right), c_{i}^{\prime} y^{\prime}\left(c_{i}^{\prime}\right)^{-1}$ is homotopic to a $c_{i} y c_{i}^{-1}$ where $c_{i}$ is a loop based at $x_{0}$. Since $[y]=\prod_{i=1}^{m}\left[\alpha_{i}\right]\left[\beta_{i}\right]\left[\alpha_{i}\right]^{-1}\left[\beta_{i}\right]^{-1}$ in $\Pi_{1}\left(X^{\prime}, x_{0}\right)$ and each $\left[\alpha_{i}\right]=\left[c_{i}\right][y]\left[c_{i}\right]^{-1}$ in $\Pi_{1}\left(M, x_{0}\right)$, we have

$$
[y]=\prod_{i=1}^{m}\left[c_{i}\right][y]\left[c_{i}\right]^{-1}\left[\beta_{i}\right]\left[c_{i}\right][y]^{-1}\left[c_{i}\right]^{-1}\left[\beta_{i}\right]^{-1}
$$

The product (1) implies $[y]$ is in the second term of the lower central series (the commutator subgroup) of $\Pi_{1}\left(M, x_{0}\right)$. Since the terms in the lower central series are normal subgroups, $\left[c_{i}\right][y]\left[c_{i}\right]^{-1}$ is also in the second term of the lower central series. Hence (1) implies $[y]$ is in the third term of the lower central series. Proceeding in this manner we see that $[y] \in\left(\Pi_{1}(M)\right)_{\omega}$.

THEOREM 1. If $K$ is of genus 1 and $l \notin\left(\Pi_{1}(C)\right)_{\omega}$, then $l(K) \neq\left(S^{1} \times S^{2}\right)^{\prime}$.

Proof. Suppose $l(K)=\left(S^{1} \times S^{2}\right)^{\prime}$. By Lemma 1 , there exists a planar surface $D$ properly embedded in $K$ such that the number of components of $\mathrm{Bd} D$ is odd, each component of $\mathrm{Bd} D$ is parallel to $K$ 's longitude $l$ and $D$ is incompressible in $K$. Put $D$ in general position relative to the minimal spanning surface $S$ of genus 1 . If any simple closed curve $x$ of $S \cap D$ bounds a disk in $S$, then, since $D$ is incompressible, $x$ bounds a disk in $D$. Suppose $x$ is innermost relative to $S$, i.e., the disk $x$ bounds in $S$ contains no points of $D$ in its interior. Then we may replace the disk $x$ bounds in $D$ by the one it bounds in $S$ and, pushing the new $D$ off $S$, we obtain a $D \cap S$ with fewer components. Since the above statements are true for $S$ interchanged with $D$, we may suppose that no simple closed curve of $S \cap D$ bounds a disk in either $S$ or $D$. If $S \cap D=\varnothing$, then $D \subset C$ and, by Lemma $2, l \in\left(\Pi_{1}(C)\right)_{\omega}$, contradicting our hypothesis. If $x$ is a simple closed curve of $S \cap D$ which separates $S$, then, since $S$ is of genus $1, x$ and $\mathrm{Bd} S$ cobound an annulus $Z$ in $S$. We may suppose Int $Z \cap D=\varnothing$. We then obtain two planar surfaces $D^{\prime}$, $D^{\prime \prime}$ by adding two copies of $Z$ to the two components of $D-x$, respectively, and adjusting by pushing both off $Z$. Now one of the resulting two surfaces $D^{\prime}, D^{\prime \prime}$, say $D^{\prime}$, has an odd number of boundary components and $D^{\prime} \cap S$ has fewer components than $D \cap S$. Suppose then that $x$ does not separate $S$ and that $x$ is innermost on $D$, i.e., one component $D^{\prime}$ of $D-x$ contains no points of $S$. Let $N(x)$ be a small regular neighborhood of $x$ in $S$. Let $S^{\prime}=S-$ Int $N(x)$ and form a new planar surface $D_{0}$ by adding two parallel copies of $D^{\prime}$ to $S^{\prime}$, i.e., fill in the two holes of $S^{\prime}$ with copies of $D^{\prime}$ to form $D_{0}$. Note that we may push $D_{0}$ off $S$ so that $D_{0} \cap S=\varnothing$ and that $D_{0}$ has an odd number of boundary components. Now in all cases we have the contradiction that $l \in\left(\Pi_{1}(C)\right)_{\omega}$ and the proof is complete.

If $F$ is a free group, then $F_{\omega}=1$ [5, pp. 311-312]; thus, we have the following corollary. 
Corollary 1. If $K$ is of genus 1 and $C$ is a cube with two handles ( $K$ has an algebraically unknotted, minimal spanning surface $)$, then $l(K) \neq\left(S^{1} \times S^{2}\right)^{\prime}$.

Corollary 1 implies that for every pretzel knot $K$ of genus 1 [7] we have $l(K) \neq\left(S^{1} \times S^{2}\right)^{\prime}$ (Theorem 1 of [6] does not apply to all pretzel knots since some have trivial Alexander polynomial.)

\section{REFERENCES}

1. P. S. Alexandrov, Combinatorial topology, Vol. 3, Graylock Press, Albany, N. Y., 1960. MR 22 \#4056.

2. R. H. Fox, A quick trip through knot theory, Topology of 3-Manifolds and Related Topics (Proc. Univ. Georgia Inst., 1961), Prentice-Hall, Englewood Cliffs, N. J., 1962, pp. 120-167. MR 25 \#3522.

3. W. Haken, Some results on surfaces in 3-manifolds, Studies in Modern Topology, Math. Assoc. Amer.; distributed by Prentice-Hall, Englewood Cliffs, N. J., 1968, pp. 39-98. MR 36 $\# 7118$.

4. William Jaco and D. R. McMillan, Jr., Retracting three-manifolds onto finite graphs, Illinois J. Math. 14 (1970), 150-158. MR 41 \# 1026.

5. W. Magnus, A. Karrass, and D. Solitar, Combinatorial group theory, Interscience, New York, 1966. MR 34 \# 7617.

6. L. Moser, On the impossibility of obtaining $S^{2} \times S^{1}$ by elementary surgery along a knot, Pacific J. Math. 53 (1974), 519-523. MR 50 \#11215.

7. K. Reidemeister, Knotentheorie, Chelsea, New York, 1948.

Department of Mathematics, University of Iowa, Iowa City, Iowa 52242 